\title{
Preliminary study of Autoimmune Polyglandular Syndrome (APS). Are they solely an endocrinological problem?
}

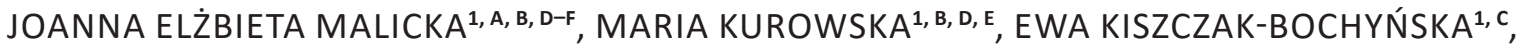 \\ MARTA DUDZIŃSKA 2, D, E, EWA MALICKA ${ }^{3, \mathrm{D}, \mathrm{E}}$, JERZY S. TARACH ${ }^{1, \mathrm{D}, \mathrm{E}}$ \\ ${ }^{1}$ Department of Endocrinology, Medical University of Lublin, Poland \\ ${ }^{2}$ Chair of Internal Medicine and Department of Internal Medicine in Nursing, Medical University of Lublin, Poland \\ ${ }^{3}$ Student of the Medical University of Lublin, Poland
}

A - Study Design, B - Data Collection, C - Statistical Analysis, D - Data Interpretation, E - Manuscript Preparation, F - Literature Search, G - Funds Collection

Summary Background. Autoimmune Polyglandular Syndrome (APS) forms a heterogeneous group of rare, genetically caused diseases, characterized by autoimmune activity against more than one endocrine organ, although non-endocrine organs can be also affected. APS was classified into four types. The two major types are APS-1 and APS-2.

Objectives. The aim of this study was to evaluate the type, number and sequence of appearance of autoimmune diseases forming different types of APS in own material.

Material and methods. The study involved 41 patients with APS (32 F; 9 M), aged 19-72 years (mean $45.2 \pm 13.5$ ). The authors analyzed medical histories and the results of additional tests.

Results. In the entire group of patients, as well as in each type of APS, women predominated. The most frequently diagnosed type of APS, confirmed in $51.2 \%$ of patients, was APS-2. APS-3 accounted for $41.5 \%$ of APS. The most rarely observed, present in $7.3 \%$ of patients, was APS-1. The most common pathology was autoimmune thyroid disease, followed by Addison's disease, and third - type 1 diabetes. The majority of patients (58.5\%) were diagnosed with only two coexisting disorders, mainly Addison's disease and thyroid disease. In $36.6 \%$ of patients, three diseases were recognized, while four autoimmune disorders were discovered in only two women (4.9\%). The onsets of consecutive autoimmune diseases in individual patients from study group were spread in time and spanned up to 29 years maximum.

Conclusions. If the patient exhibits autoimmune disorders, the suspicion of a further autoimmune disease should be present. A family history of autoimmune disease is also very helpful. A patient with APS needs life-long replacement therapy and observation by a family doctor, endocrinologist and other specialists to monitor the effectiveness of the treatment and detect new components of the syndrome. Key words: autoimmune polyglandular syndrome, APS, autoimmune diseases, Addison's disease, Hashimoto's thyroiditis.

Malicka JE, Kurowska M, Kiszczak-Bochyńska E, Dudzińska M, Malicka E, Tarach JS. Preliminary study of Autoimmune Polyglandular Syndrome (APS). Are they solely an endocrinological problem? Fam Med Prim Care Rev 2017; 19(3): 239-242, doi: https://doi. $\operatorname{org} / 10.5114 /$ fmpcr.2017.69284.

\section{Background}

The definition and first classification of Autoimmune Polyglandular Syndrome (APS) was established by Neufeld, Maclaren and Blizzard in 1980 [1]. However, researchers reported the occurrence of this type of illnesses earlier: Oegle in 1866, Parkinson in 1910, Schmidt in 1926, Rowntree and Snell in 1931, Gowen in 1932 and others [2, 3]. These syndromes form a heterogeneous group of rare, genetically caused diseases, characterized by autoimmune activity against more than one endocrine organ, although non-endocrine organs can be also affected. APS was classified into four types. The two major types of APS (APS-1 and APS-2) include primary adrenal insufficiency (Addison's disease) as a prominent component [4].

APS-1, also known as autoimmune polyendocrinopathy, candidiasis and ectodermal dysplasia (APECED), is caused by mutations of the autoimmune regulator (AIRE) gene on chromosome 21 and is inherited in an autosomal recessive manner $[5,6]$. It predominantly affects children at the age of 3 to 5 years or juvenile patients from Sardinia, Finland and Iranian Jews. The female to male ratio varies between $0.8 / 1$ and $2.4 / 1$ $[2,6]$. APS- 1 exhibits a combination of mucocutaneous candidiasis, Addison's disease and hypoparathyroidism $[5,6]$. The di- agnosis is established when two out of thee of these symptoms are present.

APS-2 is a multigenetic disorder associated with some HLA haplotypes. It is more common than APS-1 and shows a predominance in females (female/male ratio $=3 / 1$ ), without any preference of a certain ethnic group. The estimated prevalence is $\sim 1.4$ to 4.5 per 100,000 inhabitants, with peak incidence between the ages of 20 and 60 years, mostly in the third or fourth decade [2, 3]. Its main components are: Addison's disease, type 1 diabetes, autoimmune thyroid diseases (AITD) and other autoimmune disorders [5-7]. Addison's disease, as the obligatory component, is potentially life threatening [7].

APS-3 is a variant of APS-2, recognized in the case of coexistence of autoimmune thyroid disease with other autoimmune endocrinopathies other than Addison's disease. It also includes non-endocrine organ-specific autoimmune disorders, such as pernicious anemia and/or alopecia [8].

APS-4 is characterized by the occurrence of two or more organ-specific autoimmune disorders that do not meet the criteria for APS-1, APS-2 or APS-3. For example, the coexistence of Addison's disease with one or more of the diseases, other than the main APS 1, 2 and 3 components (excluding candidiasis, hypoparathyroidism, autoimmune thyroid disease and type 1 diabetes), allows for the diagnosis of APS-4 [9-11]. 


\section{Objectives}

The aim of this study was to evaluate the prevalence of different types of APS and the type, number and sequence of appearance of autoimmune diseases included in their composition.

\section{Material and methods}

The study group comprised 41 patients with APS (32 women, i.e. $78 \%$ of the study group, and 9 men, i.e. $22 \%$ of the study group), aged 19-72 years (mean $45.2 \pm 13.5$ ), hospitalized in the Department of Endocrinology of the Medical University in Lublin in the years 2011-2016. The authors analyzed medical histories and the results of additional tests on the patients.

The obtained results were subjected to statistical analysis using STATISTICA v. 10.0 software (StatSoft, Poland). The distribution of continuous variables was evaluated using the Shapiro-Wilk test. Continuous variables were expressed as mean \pm standard deviation and compared using the Student's $t$-test or Mann-Whitney U test, as appropriate. For comparing the mean of more than two groups, the ANOVA or Kruskal-Wallis test was used. Categorical variables were expressed as a number (percentages) and compared using the $\chi^{2}$ or test for two fractions. The significance level used in hypothesis testing was $5 \%$.

\section{Results}

In the entire group of 41 people, there were significantly more women than men $(p=0.0033)$. A similar relationship, without statistical significance (too few people), was found for each of the APS groups.

APS- 2 was the most frequently diagnosed type of APS. It was confirmed in 21 patients (51.2\%), including $14 \mathrm{~F}$ and $7 \mathrm{M}$. APS-3 accounted for $41.5 \%$ of APS and was detected in $17 \mathrm{pa}$ tients, including $15 \mathrm{~F}$ and $2 \mathrm{M}$. APS-1 was described most rarely and was present in 3 females (7.3\%) (Figure 1).

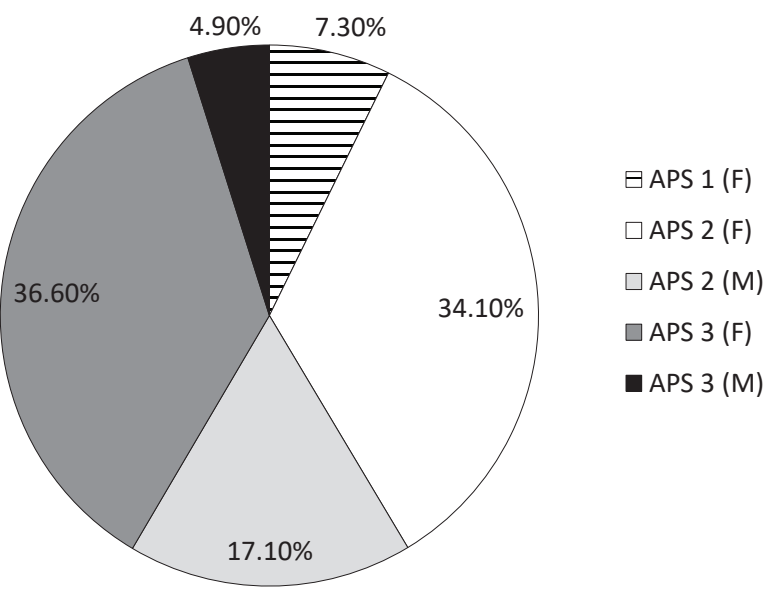

Figure 1. Frequency and type of APS in males (M) and females (F) in the study group

The most common pathology of autoimmune ground, occurring in each evaluated person, was autoimmune thyroid disease (41 cases), with 33 cases (80.5\%) of Hashimoto's disease and 8 cases $(19.5 \%)$ of Graves' disease. Hashimoto's disease was diagnosed on the basis of the presence of anti-thyroperoxidase antibodies (TPOAb), with the average value $=2,295.5$ $\mathrm{U} / \mathrm{ml}$ (range 91.0-11,100.0 $\mathrm{U} / \mathrm{ml}$; normal range 0-60 U/ml), and Graves' disease was confirmed by the presence of thyroid stimulating hormone receptor antibodies (TSHRAb), with the average value $=8.1 \mathrm{U} / \mathrm{I}$ (range $1.7-24.0 \mathrm{U} / \mathrm{I}$; normal range $<1.5$
$U / I)$. On the basis of free thyroxine (FT4), free triiodothyronine (FT3) and thyrotropin (TSH) concentrations, hormonal status was evaluated. In a case Hashimoto's thyroiditis with subclinical or overt hypothyroidism (27 cases), the patient was receiving adequate substitution therapy with levothyroxine. 6 patients were euthyroid. Graves' thyrotoxicosis was treated with tiamazole, ${ }^{131}$ I or surgically.

Primary adrenal insufficiency (Addison's disease) was present in each case of APS-2 and APS-1 (24 patients, 58.5\%) and was the second most common pathology. It was confirmed by the presence of low serum cortisol concentrations (range: 0.2-9.3 $\mu \mathrm{g} / \mathrm{dl}$; mean value: $2.5 \mu \mathrm{g} / \mathrm{dl}$; normal range: 4.3-22.4 $\mu \mathrm{g} / \mathrm{dl}$ ) and high adrenocorticotropin (ACTH) concentrations (range: $142.2-5,620.0 \mathrm{pg} / \mathrm{ml}$; mean value: $1,368.5 \mathrm{pg} / \mathrm{ml}$; normal range: $7.2-63.6 \mathrm{pg} / \mathrm{ml}$ ). All patients were administered adequate substitution treatment.

Type 1 diabetes was statistically significantly less frequent in APS-2 compared to APS-3 ( $p=0.0014)$ and occurred in 16 patients (39\%): in 4 with APS-2 and in 12 with APS-3. All 3 cases of APS-1 presented the coexistence of Addison's disease, Hashimoto's disease and candidiasis. Primary hypoparathyroidism was observed in only 1 case of APS-1, but also in 1 case of APS- 2 and APS-3.

Among non-endocrine affected organs were: vitiligo in 5 patients (12.2\%): 3 cases with APS-3 and 2 cases with APS-2, pernicious anemia in 4 patients $(9.8 \%)$ with APS-3, autoimmune hepatitis - in 2 APS-2 patients (4.9\%), celiac disease in 1 female with APS-2, psoriasis and psoriatic arthritis - in 1 female with APS-2, systemic sclerosis - in 1 male with APS-3, and systemic lupus erythematosus - in 1 female with APS-3.

The majority of patients (24 patients $=58.5 \%$ ), including 13 with APS-2 (31.7\%) and 11 with APS-3 (26.8\%), were diagnosed with only two coexisting disorders, mainly Addison's disease and autoimmune thyroid disease. In 15 (36.6\%) patients: 7 with APS-2 (17.1\%), 6 with APS-3 (14.6\%) and 2 with APS-1 (4.9\%), three diseases were recognized, while four autoimmune disorders were discovered only in 2 women (4.9\%): 1 with APS-2 (Addison's disease, Hashimoto's hypothyroidism, type 1 diabetes and hypoparathyroidism) and 1 with APS-1 (Addison's disease, Hashimoto's hypothyroidism, hypoparathyroidism and mucocutaneous candidiasis) (Figure 2).

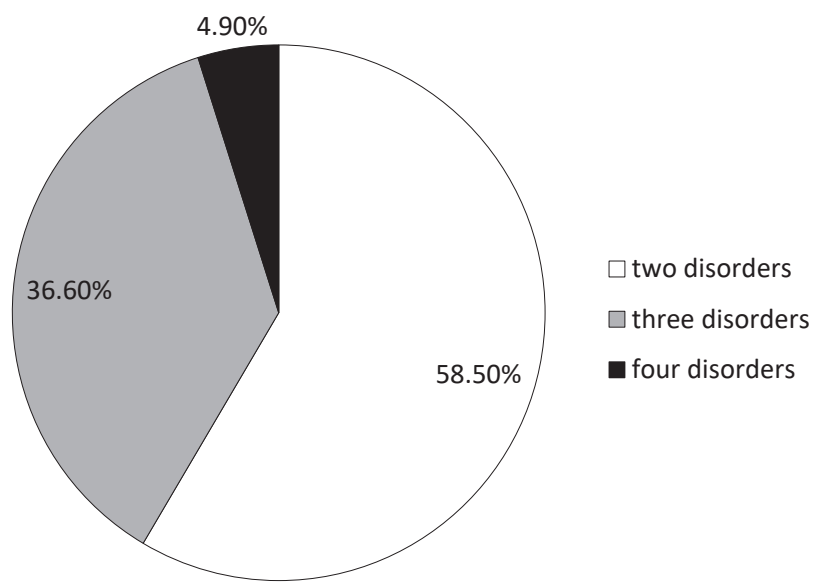

Figure 2. Number of coexisting disorders in our patients with APS

The onsets of consecutive autoimmune diseases in individual patients from study group were spread in time and spanned up to 29 years maximum.

\section{Discussion}

Thyroid disorders, which are the most frequent expression of adult APS $[11,12]$, were also dominant in our group and oc- 
curred in each patient. We also observed autoimmune thyroiditis in APS-1 patients, although other authors report that such disorders are, surprisingly, rare in APS-1 [11].

We recognized Hashimoto's thyroiditis in $80.5 \%$ - 33 patients (predominantly with subclinical and overt hypothyroidism - 27 cases, asymptomatic thyroiditis -6 cases) and Graves' disease in $19.5 \%-8$ patients (in 3 with orbitopathy), more often than other authors. In 360 patients with APS-2, presented by Wémeau et al. [11], AITD was confirmed in various expressions in $66 \%$ of patients: as thyroid atrophy, hypertrophic goiter related to Hashimoto's thyroiditis, Graves' disease, asymptomatic autoimmune thyroiditis, etc. Förster et al. [12] reported hyperthyroidism in 33\% out of 151 APS-2 patients, Hashimoto's thyroiditis in $32.5 \%$ and positive thyroid-associated antibodies without manifest signs of thyroid disease in $52 \%$.

Referring to Wémeau et al. [11], the thyroid hormonal function may adversely affect the course of the associated disorders. Thus, an assessment of the severity of hyper or hypothyroidism may help in evaluating the therapeutic options towards the coexisting endocrine pathologies.

Addison's disease was the second most common pathology in our group ( 24 cases $=58.5 \%$ ), while its frequency varies in different reports: $18.5-19 \%$ in APS-2 $[11,12]$ and $22-80 \%$ in APS-1 [11]. It is well known that this disease is potentially life threatening; therefore, early suspicion of it is extremely important. The role of the family doctor in this case is crucial, because it often depends on the doctor how much time will pass before the patient begins effective treatment [13]. It is important that the family doctor is familiar with the symptoms of Addison's disease and takes into account adrenal insufficiency in the differential diagnosis of chronic fatigue, syncope, decrease of blood pressure, hypoglycemia (e.g. unexplained hypoglycemia in type $1 \mathrm{di}-$ abetes), especially when darkening of the skin coexists [13]. The next step is to confirm the diagnosis and to refer the patient to an endocrinologist, but the family doctor cannot delay the start of treatment due to the diagnostic process. A very important role is also played by nonpharmacological treatment: education of the patient and his/her family on how to adapt treatment to stressful situations in order to avoid adrenal crisis. The family doctor, who accompanies the patient in everyday life, should foresee the situations that require intensive supervision and treatment and consider the possibility of a new autoimmune disease in the case of new complaints or symptoms [13,14].

We recognized type 1 diabetes in 16 patients (39\%): in 4 with APS-2 and in 12 with APS-3. It was the third most common pathology in our group, although occurring less frequently than was reported in literature. According to Wémeau et al. [11], type 1 diabetes occurs in $4-12 \%$ patients with APS- 1 and in $61 \%$ with APS-2, and as reported by Förster et al. [12], also in $61 \%$ cases of APS-2.

The most common combination of autoimmune diseases in our group was Addison's disease with AIDT (13 patients = $31.7 \%$ ), while other authors predominantly observed the coexistence of type 1 diabetes with AITD: Förster et al. 33\% [12], Kahaly $41 \%$, who also reported the coexistence of AITD and Addison's disease (Schmidt syndrome) in 15\% [15].

Source of funding: This work was funded by the authors' resources. Conflict of interest: The authors declare no conflict of interests.

\section{References}

1. Neufeld M, Maclaren NK, Blizzard RM. Autoimmune polyglandular syndromes. Pediatric Annals 1980; 9: 154-162.

2. Van den Driessche A, Eenkhoorn V, Van Gaal L, et al. Type 1 diabetes and autoimmune polyglandular syndrome: a clinical review. Neth J Med 2009; 67(11): 376-387.

3. Betterle C, Lazzarotto F, Presotto F. Autoimmune polyglandular syndrome type 2: the tip of an iceberg? Clin Exp Immuno/ 2004; 137(2): 225-233, doi: 10.1111/j.1365-2249.2004.02561.x.

4. Cutolo M. Autoimmune polyendocrine syndromes. Autoimmun Rev 2014; 13(2): 85-89.

5. Komminoth P. Polyglandular autoimmune syndromes: an overview. Pathologe 2016; 37(3): 253-257, doi: 10.1007/s00292-016-0159-7.
It is well recognized that the time between manifestations of each disease is highly variable, and more than 20 years may elapse between the onset of one endocrinopathy and the diagnosis of the next [4]. The occurrence of subsequent autoimmune diseases in our group was extended in time and reached a maximum of 29 years. Therefore, it is clear that patients need life-long monitoring and observation. The key role in this area is played by the primary care doctor. Such recommendations, e.g. regarding the screening of patients with diabetes towards the development of AITD, are also provided by other authors [16] and scientific societies, such as the Polish Society of Diabetology [17].

Autoimmune endocrinopathies are sometimes associated with non-endocrine autoimmunopathies. In our cohort, we confirmed a total of 15 of such diseases. The most common was vitiligo (5 cases $-12.2 \%$ ), but its frequency was lower than in other observations, e.g. $20 \%$ in APS-2, as reported by Förster et al. [12] or Wémeau et al. [11].

Pernicious anemia was present in 4 of our patients with APS-3 (9.8\%). It was also described in coexistence with Addison's disease and primary hypothyroidism by Vrkljan et al. [18], who emphasizes the link between APS-2 and APS-3, referring to etiological factors, as well as pathophysiological and clinical aspects. Pernicious anemia was also present in $5 \%$ of APS- 2 patients evaluated by Förster et al. [12] and in 5.3\% of APS-2 patients evaluated by Wémeau et al. [11].

Autoimmune hepatitis and celiac disease are rarely recognized in APS $[10,19,20]$. We found autoimmune hepatitis in 2 patients $(4.9 \%)$ and celiac disease in only 1 female.

It has been proven that APS may coexist with connective tissue diseases [21]. We also recognized 1 case from each of the following diseases: psoriasis and psoriatic arthritis, systemic sclerosis and systemic lupus erythematosus.

Hypoparathyroidism rarely occurs without mucocutaneous candidiasis [22], but there were 2 such cases in our group.

Surprisingly, unlike the other researchers $[11,12]$, we did not record any cases of hypogonadism or alopecia. The limitation of our study is the relatively small group of patients, associated with the rare prevalence of APS in the general population. Despite this, our observations, similarly to the findings of the previously cited authors, confirm the complexity of the problem arising from the diversity of diseases forming these syndromes, their appearance over many years and involvement in the care of the patients

\section{Conclusions}

If the patient exhibits one, two or more autoimmune disorders, especially Addison's disease, Hashimoto's thyroiditis or type 1 diabetes, a high index of suspicion for a further autoimmune disease should be present. A family history of autoimmune disease is also very helpful in the evaluation of such cases. Patient with APS needs life-long replacement therapy and observation by a family doctor, endocrinologist and other specialists to monitor the effectiveness of the treatment and detect new components of the syndrome. themselves, their families and doctors of various specialties. 
6. Lankisch TO, Jaeckel E, Strassburg CP, et al. Autoimmune polyglandular syndromes. Internish (Berlin) 2005; 46(7): 750-758.

7. Wang $X$, Ping F, Qi C, et al. Delayed diagnosis with autoimmune polyglandular syndrome type 2 causing acute adrenal crisis: a case report. Medicine (Baltimore) 2016; 95(42): e5062.

8. Makino S, Uchihashi T, Kataoka Y, et al. Recovery from alopecia areata in a patient with autoimmune polyglandular syndrome type 3. Endocrinol Diabetes Metab Case Rep 2015: 140084, doi: 10.1530/EDM-14-0084.

9. Betterle C, Garelli S, Coco G, et al. A rare combination of type 3 autoimmune polyendocrine syndrome (APS-3) or multiple autoimmune syndrome (MAS-3). Auto Immun Highlights 2014; 5(1): 27-31, doi: 10.1007/s13317-013-0055-6.

10. Lakhotia M, Pahadia HR, Kumar H, et al. A case of Autoimmune Polyglandular Syndrome (APS) type II with hypothyroidism, hypoadrenalism, and celiac disease - a rare combination. J Clin Diag Res 2015; 9(4): OD01-OD03, doi: 10.7860/JCDR/2015/10755.5748.

11. Wémeau JL, Proust-Lemoine E, Ryndak A, et al. Thyroid autoimmunity and polyglandular endocrine syndromes. Hormones (Athens) 2013 ; 12(1): 39-45.

12. Förster G, Krummenauer F, Kühn I, et al. Polyglandular autoimmune syndrome type II: epidemiology and forms of manifestation. Dtsch Med Wochendchr 1999; 124(49): 1476-1481.

13. Zdrojowy-Wełna A, Bednarek-Tupikowska G. Postępowanie w przewlekłej niewydolności kory nadnerczy - rola lekarza rodzinnego. Fam Med Prim Care Rev 2013; 15(3): 486-490 (in Polish).

14. Fichna M, Fichna P, Gryczyńska M, et al. Screening for associated autoimmune disorders in Polish patients with Addison's disease. Endocrine 2010; 37(2): 349-360, doi: 10.1007/s12020-010-9312-x.

15. Kahaly GJ. Polyglandular autoimmune syndromes. Eur J Endocrinol 2009; 161: 11-20.

16. Szcześniak G, Zdybel W, Kozak-Nurczyk P, et al. An evaluation of the prevalence of autoimmunological diseases in patients with diabetes and obesity hospitalized in the Department of Diabetology, Rural Medicine Institute in Lublin. Fam Med Prim Care Rev 2016; 18(3): 345-347.

17. PTD. Zalecenia kliniczne dotyczące postępowania u chorych na cukrzycę 2017. Diabetol Prakt 2017; 3(Supl. A): A73 (in Polish).

18. Vrkljan AM, Pašalić $A$, Strinović $M$, et al. Coexistence of Addison's disease and pernicious anemia: is the new classification of autoimmune polyglandular syndrome appropriate? Acta Clin Croat 2015; 54(2): 232-235.

19. Sagar A, Valson A, Bhartiya M. Autoimmune polyglandular syndrome type II: Schmidt's syndrome, a unifying diagnosis in a case presenting with an uncommon combination of multiple endocrine disorders. Indian J Endocrinol Metab 2016; 20(4): 579-580, doi: 10.4103/2230-8210.183477.

20. Dieli-Crimi R, Núñez C, Estrada L, et al. An autoimmune polyglandular syndrome complicated with celiac disease and autoimmune hepatitis. Ann Hepatol 2016; 15(4): 588-591.

21. Wielosz E, Majdan M, Kurowska M, et al. Autoimmune polyglandular syndromes - literature review and analysis of clinical course in chosen cases. Wiad Lek 2016; 69(1): 27-32.

22. Krysiak R, Kobielusz-Gembala I, Okopień B. Atypical clinical presentation of autoimmune polyglandular syndrome type 4. Przegl Lek 2011; 68(6): 339-341.

Tables: 0

Figures: 2

References: 22

Received: 28.03.2017

Revised: 07.06.2017

Accepted: 09.06.2017

Address for correspondence:

Joanna Elżbieta Malicka, MD, PhD

Klinika Endokrynologii SPSK4

ul. Jaczewskiego 8

20-954 Lublin

Polska

Tel.: +48 81 724-46-68

E-mail: malickajoanna@interia.pl 